Annuaire du Collège de France 2017-2018

\title{
Architecture et forme urbaine
}

Jean-Louis Cohen

\section{OpenEdition}

Journals

Édition électronique

URL : https://journals.openedition.org/annuaire-cdf/15241

DOI : 10.4000/annuaire-cdf. 15241

ISBN : 978-2-7226-0572-5

ISSN : 2109-9227

Éditeur

Collège de France

Édition imprimée

Date de publication : 30 décembre 2020

Pagination : 539-550

ISBN : 978-2-7226-0516-9

ISSN : 0069-5580

Référence électronique

Jean-Louis Cohen, «Architecture et forme urbaine », L'annuaire du Collège de France [En ligne], 118| 2020, mis en ligne le 01 avril 2021, consulté le 22 août 2022. URL : http://journals.openedition.org/ annuaire-cdf/15241 ; DOI : https://doi.org/10.4000/annuaire-cdf.15241 


\title{
ARCHITECTURE ET FORME URBAINE
}

\author{
Jean-Louis COHEN
}

Architecte et historien, professeur en histoire de l'architecture, titulaire de la chaire Sheldon $\mathrm{H}$. Solow à l'Institute of Fine Arts, New York University (États-Unis), professeur invité au Collège de France

Mots-clés : architecture, urbanisme, arts, ville, politique, nazisme, stalinisme, démocratie

La série de cours « L'architecture, vecteur du politique » est disponible en audio et en vidéo, sur le site internet du Collège de France (https://www.college-de-france.fr/ site/jean-louis-cohen/course-2017-2018.htm), ainsi que le colloque « Architectures de la politique, politiques de l'architecture » (https://www.college-de-france.fr/site/jeanlouis-cohen/symposium-2017-2018.htm).

\section{ENSEIGNEMENT}

\section{COURS - L'ARCHITECTURE, VECTEUR DU POLITIQUE : LE GOUVERNEMENT} DE L'ESPACE

Les relations entre l'architecture et la politique ont trop souvent été réduites au lien direct entre les gouvernants, et notamment les dictateurs, pour ce qui est du $\mathrm{XX}^{\mathrm{e}}$ siècle, et les concepteurs. Pourtant, l'espace dans lequel l'architecture, définie à la fois comme culture et comme pratique professionnelle, interagit avec le politique n'est pas plus isotrope qu'il n'est homogène.

Il serait plutôt, pour le dire avec Michel Foucault, façonné par cette « microphysique du pouvoir », selon laquelle la domination politique opère au travers de réseaux instables d'actions. Parmi ces réseaux, la forme urbaine et l'architecture sont incontestablement sujettes à la pression des pouvoirs politiques, mais elles n'en sont pas moins déterminées par les forces du marché et par les attentes des groupes sociaux concurrents.

Tout en faisant office de mode de représentation au travers de ses productions monumentales, l'architecture donne forme aux relations quotidiennes à toutes les échelles du tissu social. Le délicat ajustement entre le répertoire des formes disponibles et les attentes des différentes composantes de la société s'opère selon 
une matrice dans laquelle discours et pratiques se croisent, les architectes étant parfois des protagonistes dont le programme s'impose aux politiques. Un large ensemble de projets et d'édifices condensant ces enjeux, des Amériques à l'Asie, en passant par l'Europe occidentale et orientale et l'Afrique du Nord, est mis en perspective dans le cours et le colloque qui le prolonge.

\section{Cours 1 - Architecture des pouvoirs et pouvoir des architectes}

Les récits rendant compte des relations entre les langages architecturaux et les régimes politiques portent notamment sur les découpages temporels appliqués à l'architecture, sur lesquels ont été plaqués ceux qui correspondent aux principales ruptures dans l'ordre de la politique et non ceux qui correspondent aux seuils dans les transformations des discours et des formes.

Ces découpages sont remplacés par un nouveau cadre d'interprétation qui conduit à une cartographie alternative des intersections entre la sphère politique et celle de l'architecture moderne et contemporaine. Celle-ci s'établit au travers de l'étude des trajectoires parallèles d'architectes engagés dans la conception de projets importants pour des dirigeants politiques ou des entrepreneurs, ce qui permet d'apprécier autrement les pressions exercées d'en haut, en quelque sorte, sur les experts, tout comme la capacité de ceux-ci à y résister ou à les subvertir, et leur efficacité à obtenir le soutien de ces forces à leurs projets.

De nombreux professionnels de qualité et/ou de renom ont utilisé leurs relations avec le pouvoir pour atteindre leurs propres objectifs culturels et esthétiques, surtout lorsque leurs carrières furent longues. Cela fut le cas tant des conservateurs - hostiles à la modernité, mais non à la modernisation - que des modernes des différentes obédiences, des plus attachés à une certaine tradition aux plus radicaux. Ce faisant, tous aspiraient à augmenter leur capital matériel et leur capital symbolique, si l'on peut transposer à la sphère architecturale le concept proposé par Pierre Bourdieu. Les architectes ont manipulé et influencé les clients appartenant à des régimes politiques changeants, réussissant en définitive à former des œuvres qui transcendent les limites mêmes de la politique.

\section{Cours 2 - Représenter le temps : changements et ruptures}

L'un des terrains de rencontre les plus spectaculaires entre l'architecture et la politique se forme lors de la mise en place d'un nouvel ordre social, consécutif à une victoire militaire ou à une révolution. Des édifices éphémères sont dressés pour célébrer le changement, dont certains prennent une forme permanente. Les parades et les manifestations définissent de nouvelles topographies urbaines, elles aussi destinées souvent à perdurer dans la forme des villes.

Dans le même temps, une architecture en quelque sorte négative se manifeste avec le vandalisme des nouveaux pouvoirs, prompts à effacer les traces bâties des régimes antérieurs.

De nombreux projets engagés depuis la fin du XIX ${ }^{\mathrm{e}}$ siècle pour manifester avec des édifices ou des tracés urbains la force des régimes émergents peuvent être placés à l'enseigne de l'œuvre d'art totale, telle que l'a définie Richard Wagner, opérant à des échelles multiples et avec des médias distincts, mais partie prenante d'un même chœur architectural. 
L'analyse porte sur les épisodes relatifs à la Révolution française et à l'Empire, aux révolutions russe et allemande, au fascisme italien et au $\mathrm{III}^{\mathrm{e}}$ Reich. Dans la sphère coloniale, où la mise en scène de la domination a largement utilisé le registre de l'architecture, l'attention porte sur des villes comme Alger ou Casablanca, les formes de la rupture coloniale participant par ailleurs d'une réappropriation des dispositifs existants, dont des interventions à portée symbolique transforment le sens.

\section{Cours 3 - L'expérience du temps et les paradoxes de la continuité}

Lorsque les régimes politiques entendent fonder leur légitimité sur l'évocation bâtie de moments historiques révolus, leur rapport au passé prend des formes particulières, notamment lorsqu'il s'applique à deux villes distantes dans l'espace et dans le temps. Il ne s'agit plus alors de l'identification à un état antérieur d'un lieu, mais plutôt d'un double transfert par lequel une forme urbaine en vient à en contenir une autre, selon un principe homologue de celui de l'intertextualité dans le champ littéraire, que l'on peut dénommer « interurbanité ». Comme dans les relations entre deux textes, celles qui sont établies entre deux formes urbaines peuvent procéder par citation, paraphrase, parodie, inclusion, condensation, et selon de multiples autres figures - comme c'est le cas d'ailleurs pour les relations entre édifices. Il en va ici de ce que Gérard Genette dénomme l' « architexte », qu'il définit comme « cette relation d'inclusion qui unit chaque texte aux différents types de discours auxquels il ressortit». La fortune sémantique et concrète des éléments de la forme urbaine de Rome, du Forum au Capitole, en est un bon exemple.

Le dispositif de la translation d'une forme dans le temps - une des formes de l'intertextualité architecturale - porte selon les cas et parfois simultanément, si l'on utilise un modèle linguistique, sur la syntaxe et sur le lexique. Le recours à ce dernier est le plus évident, tant il est facile de réduire l'histoire de l'architecture à celle de «styles » dont le décor serait l'expression, et qu'il serait possible d' «appliquer » à des formes spatiales différentes. Mais la syntaxe, autrement dit le mode d'organisation des espaces et des éléments d'architecture, peut aussi faire l'objet d'une appropriation.

Tout se passe donc comme si la pulsion des architectes au réexamen - allant jusqu'à la réplique - était largement partagée par les régimes à la recherche de fragments d'histoire disponibles, fragments dans lesquels les formes architecturales sont invariablement couplées avec des récits rassurants ou mobilisateurs. Le plus souvent mythiques, ces récits partagent, en dépit de leurs différences, un même centrage sur un âge d'or, dont le retour serait rendu possible par le truchement magique de l'architecture.

\section{Cours 4 - Épisodes allemands, de Weimar à la guerre froide}

Deux coupes longues rendant compte de l'interaction entre politique et architecture en Allemagne peuvent être tracées. La première fait apparaître l'enchaînement assez rapide des régimes politiques entre l'unité allemande de 1871 et la fin de la guerre froide que marque la Wende, intervenue en 1989-1990. La seconde révèle les profondes transformations de la théorie et de la pratique de l'architecture pendant ces 120 années, informées par celle des sciences, de la culture et des arts. Des coupes plus courtes sont indispensables pour mesurer la dynamique plus interne de l'architecture, en révélant les péripéties constitutives des trajectoires de plusieurs 
professionnels dont la production a traversé les régimes, qu'il s'agisse des activités menées dans les années 1920 et 1930 par ceux qui furent formés avant 1914, de celles qui furent menées sous le nazisme par les architectes formés sous Weimar, ou encore de celles, plus tendues, des architectes dont la pratique s'est déployée au cours de quatre régimes successifs. Latéralement, les mouvements de certains entre les deux Allemagne, les exils et les retours doivent aussi être pris en compte.

L'analyse de la longueur des trajectoires des architectes et de leur capacité à traverser les régimes sans nécessairement infléchir leur problématique éclaire cette configuration. Sont donc considérés les parcours d'une vingtaine d'individus nés entre la fin des années 1860 et le début du XXe siècle, dans leur intersection avec les régimes politiques successifs sous lesquels ils ont opéré. L'analyse porte ainsi sur les trajectoires de figures déterminantes de la culture architecturale allemande que furent Peter Behrens, Hans Poelzig, Paul Schultze-Naumburg, Heinrich Tessenow, Paul Schmitthenner, Paul Bonatz, Wilhelm Kreis, Hans Scharoun, Ernst May, Bruno Taut, Ludwig Mies van der Rohe et Egon Eiermann.

\section{Cours 5 - Figures italiennes, du futurisme au postmodernisme}

Les péripéties et les caractères de la politique italienne depuis la seconde moitié $\mathrm{du} \mathrm{XIX}^{\mathrm{e}}$ siècle partagent quelques traits communs avec l'Allemagne, tels que l'alternance de régimes opposés et la diversité régionale. Comme en Allemagne, l'exercice du pouvoir d'État central doit compter avec des configurations locales fortes, dont l'autonomie est notable dans les champs de la culture et de l'architecture. Deux coupes diachroniques sont éclairantes, la première portant sur les régimes successifs et les configurations des villes et de la culture, et la seconde sur les itinéraires des architectes et leurs rencontres avec les pouvoirs, notamment ceux des protagonistes ayant eu les trajectoires les plus longues.

Sous le fascisme, les rivalités et les divergences entre architectes et urbanistes ne font que réverbérer celles qui opposent les hiérarques du régime, au sein duquel l'aile corporatiste soutient les projets des modernes. Ces tensions affectent jusqu'aux périphéries de l'Empire colonial italien. Comme dans le cas de l'Allemagne, des groupes se détachent, que l'on peut définir en croisant la démographie, les expériences politiques au travers des régimes successifs, les expériences institutionnelles et professionnelles et l'ancrage régional.

La plus originale des figures apparaissant au sein de ces groupes est celle de Marcello Piacentini, actif pendant six décennies, au cours desquelles son rôle politique fut considérable, grâce à sa capacité d'adaptation hors pair. Visiteur fréquent de Mussolini, manipulant concours et appels d'offres, il réalisa des rénovations urbaines marquantes à Brescia et à Gênes. Loin de s'opposer frontalement aux jeunes modernes, il en récupéra l'énergie, et s'appropria l'aura de la nouvelle production mondiale. Pivot de ces deux entreprises déterminantes du régime que sont la Cité universitaire et l'E42, dans lesquelles il sut assimiler les nouveaux langages, il parvint à être blanchi en 1945 et à rester engagé dans les opérations romaines jusqu'à sa disparition.

\section{Cours 6 - Les affects politiques de Le Corbusier}

Figure exceptionnelle de l'architecture du $\mathrm{XX}^{\mathrm{e}}$ siècle, Le Corbusier l'est moins du fait de ses positions politiques que par la contribution que ses théories et ses projets ont apportée à la pensée sur la ville et les édifices. Parallèlement, il n'a cessé de 
solliciter l'opinion publique et de s'adresser aux décideurs publics en les sommant d'adopter ses plans.

L'analyse des conjonctures de son rapport à la politique met en évidence des positions récurrentes. La première est tout simplement la dénégation, l'affirmation répétée que la politique ne l'intéresse pas. S'il professe une phobie manifeste pour la politique, son intérêt latent est soutenu. Il ne cesse en fait de se situer en fonction des rapports de force du moment, tandis qu'il est lui-même annexé par les forces politiques.

La campagne de relations publiques qu'il engage en 1920 en publiant L'Esprit nouveau, puis en présentant ses projets urbains sur la scène parisienne, fait de lui une figure publique que la presse ne peut ignorer. Chacune des configurations de ses affects politiques doit donc être lue selon deux perspectives - celle des auteurs qui le critiquent ou l'encensent, et celle selon laquelle il s'approprie le discours de ses interlocuteurs politiques ou les met en demeure de le suivre.

Il y a en effet une dimension discursive dans l'ensemble de ces échanges, au travers desquels Le Corbusier intériorise et reformule les thèmes et le lexique des politiques, leur tendant comme un miroir architectural de leur programme, sans pour autant infléchir le moins du monde la substance de ses projets: il sera plus bolchevique que les Russes, plus pétainiste que le Maréchal et plus démocratechrétien qu'Eugène Claudius Petit.

La longue série de ces échanges est retracée, de ses sympathies socialistes des débuts à son flirt poussé avec les technocrates des années 1930, son intérêt pour le Front populaire, ses tentations de la période de Vichy à ses professions de foi pacifistes de l'après-guerre.

\section{Cours 7 - La démocratie comme maître d'ouvrage}

Dans un aphorisme bien connu, Henri Lefebvre a affirmé que «la ville était la projection au sol des rapports sociaux ». Que se passe-t-il si l'on falsifie cette affirmation pour dire qu'elle est la «projection au sol des rapports politiques »? Comment saisir les liens reliant les configurations du politique à ses différentes échelles, et le dessin de la ville et de ces édifices, souvent indissociables, tant il est vrai que les bâtiments publics ont, par leur implantation et les espaces qui les entourent, une empreinte dépassant celle de la trace au sol de leurs murs?

Cette question est particulièrement complexe, dès lors qu'elle porte sur les processus démocratiques et leur interaction avec les programmes d'aménagement et de construction.

Dans le cycle de la reconstruction de l'Europe occidentale après 1945, la démocratie a ainsi contribué à faire échouer certains projets, comme celui de Marcel Lods à Mayence. Elle a par la suite été présentée comme force positive. Un des propos les plus marquants dans cette perspective reste celui tenu en 1960 lors de l'inauguration de la Philharmonie de Hans Scharoun par le député social-démocrate Adolf Arndt, qui déclara alors : "la démocratie [en] est le maître d'ouvrage ». Ce slogan a présidé à nombre de discussions engageant les élus de l'Allemagne fédérale, puis de l'Allemagne réunifiée, avec des effets architecturaux marqués et marquants - positifs autant que négatifs pour ce qui est de la définition et de la réalisation des projets.

Deux matrices doivent être distinguées ici : celle de la représentation élue et celle de l'expression directe des destinataires, dont les partisans sont évidemment animés par les meilleures intentions démocratiques. Dans les opérations engagées par les représentants du peuple, les architectes sont en quelque sorte leurs délégués, ils 
donnent une forme bâtie à leur programme, au travers de diverses médiations. Dans les opérations engagées dans un cadre participatif, les architectes sont au contraire les agents des habitants, dans un contact direct avec chacun d'entre eux, auxquels ils donnent à l'occasion une réelle capacité à configurer l'espace de leur vie quotidienne.

\section{Cours 8 - Générations et expériences partagées}

La configuration spécifique du rapport entre l'architecture et le politique, tel qu'il est façonné par l'action de groupes précis, de la fin du XVIII ${ }^{\mathrm{e}}$ siècle à nos jours, peut être examinée en interrogeant la notion de génération. Après Karl Mannheim, l'une des réflexions les plus fécondes pour saisir leur jeu est celle que l'historien Reinhart Koselleck a proposée dans son Futur passé ${ }^{1}$. Si l'on s'inscrit dans la perspective qu'il trace, on pourrait dire que chaque génération partage tant un même « horizon d'attente » qu'un même « espace d'expérience ».

Une série d'épisodes considérés auparavant de façon longitudinale - ou diachronique - sont donc revisités de façon synchronique. Politique plus qu'architecturale, l'entrée correspond bien souvent aussi à des seuils dans la théorie et la pratique de l'architecture. Ces seuils coïncident évidemment avec la liquidation, puis le remplacement des hiérarchies et des institutions existantes, qui sont souvent la conséquence des mutations révolutionnaires et l'apparition de nouvelles formations et espérances sociales. Ils s'inscrivent aussi dans cet horizon d'attente que l'on nomme « progrès ». Plus précisément, ils jalonnent l'émergence de la modernité, horizon d'attente détaché des expériences antérieures. Sont ainsi interrogées dans leurs attentes et leurs expériences les générations de 1789, de 1848, de 1871, de 19141918, de 1933, de 1940-1945, de 1968, et les contemporains.

Cette étude synchronique des générations, qui reste largement à faire, comme celle, diachronique, des trajectoires longues des architectes, des décideurs et des militants, est indispensable à la compréhension des ajustements entre architecture et politique.

\section{Colloque - ARChitectures de LA POLITIQUe, POLITIQUeS DE L'ARCHITECTURE}

Inscrites dans le prolongement du cours «L'architecture, vecteur du politique : le gouvernement de l'espace», les contributions de ce colloque s'étant déroulé le 15 juin 2018 au Collège de France explorent un ensemble d'épisodes lors desquels le jeu entre dirigeants politiques et architectes s'est noué. Les interventions dont le fil conducteur est constitué, selon les cas, par la trajectoire des architectes ou par celle des politiques sont croisées avec celles qui proposent l'interprétation d'une conjoncture précise de la relation entre les deux sphères.

\section{Introduction aux débats}

\section{Jean-Louis Cohen}

La relation entre architecture et politique ne peut être réduite à la rencontre entre les individus, par exemple les dirigeants charismatiques ou autoritaires et les «grands »

1. Reinhart KoselleCK, Le Futur passé. Contribution à la sémantique des temps historiques, traduit de l'allemand par J. HoocK et M.-C. HoocK, Paris, Éditions de l'EHESS, 1990. 
architectes, mobilisant des agents de rang inférieur dans les deux domaines. Elle se déploie à des échelles très différentes, du plan de ville, à commencer par celui des capitales existantes ou créées, aux aménagements les plus ténus. L'enjeu de la représentation des pouvoirs est déterminant: dans le cas de la représentation du changement, de l'avènement d'un régime, ou de sa chute, c'est la rupture qu'exprime l'architecture; dans le cas de la mise en scène des précédents historiques, c'est la continuité avec des âges d'or mythifiés que l'architecture souligne.

Des programmes tendent précisément à la représentation, notamment ceux des régimes totalitaires et autoritaires, pour lesquels le choix des architectes est souvent effectué au terme de concours à grand spectacle (Moscou, Rome). Les programmes des démocraties utilisent fréquemment la même technique de recrutement, au service de projets aussi monumentaux. Mais la relation à la politique est également faite d'ajustements plus capillaires entre citoyens, utilisateurs, usagers ou habitants, d'une part, et concepteurs, de l'autre - ajustements opérés au travers de structures délibératives ou participatives.

$\mathrm{Au}$ demeurant, les lieux du pouvoir sont loin d'être les seules expressions du politique. En utilisant à la marge les concepts de Marx, les politiques portant sur les grands équipements contribuent à constituer le capital fixe social - l'espace bâti. Et les politiques conduites dans le champ de l'habitation, de l'école ou de la santé permettent la reproduction de la force de travail. Ces politiques trouvent des manifestations architecturales changeantes, et des esthétiques paradoxales, les plus modernes n'étant pas nécessairement les plus radicales. La dimension du temps est déterminante et peut être saisie de multiple manière. Les architectes dont l'activité s'inscrit dans une trajectoire longue - plusieurs décennies parfois - traversent des régimes différents, et tendent à les utiliser au service de leur stratégie de projet, plus qu'ils ne sont utilisés par eux.

Les figures se dégageant des analyses possibles sont multiples, car les protagonistes sont des humains dont les actions sont chargées psychologiquement. Les attitudes vis-à-vis des pouvoirs génèrent ainsi espoirs, illusions et frustrations, selon qu'un projet est accepté, refusé, ou corrompu par l'intervention des politiques. Face à cette intervention, les réponses vont de l'intransigeance à l'accommodement - plus ou moins servile -, en passant par l'intériorisation de la contrainte. Contrairement à la poésie et à la peinture, que l'exil, qu'il soit extérieur ou intérieur, permet de pratiquer comme une forme de résistance, l'architecture peut se réfugier dans une pratique intime de projets idéaux ou inscrits dans un avenir encore brumeux. Mais elle reste dans l'ensemble dépendante de moyens matériels dont seuls les appareils d'État et les entreprises disposent. Elle constitue de ce fait une délégation de pouvoir.

\section{L'architecte anticipateur du politique : Athènes 1833}

\section{Yannis Tsiomis (architecte, directeur d'études à l'EHESS)}

Lors de la fondation d'Athènes, ville-capitale de l'État néohellénique, le rapport intime entre le politique et l'architecture peut être décomposé en trois temps. Le premier est celui de l'anticipation, par des architectes, du choix et du plan d'Athènes, avant même qu'une décision politique soit prise. Le deuxième, qui s'étend jusqu'au milieu du $\mathrm{XX}^{\mathrm{e}}$ siècle, est celui de l'inversion, le politique prenant l'initiative et déformant ou reformant le plan de la ville au travers de l'intrusion de nouveaux acteurs - forces économiques, habitants, etc. Pendant ce temps, la capitale joue 
encore son rôle emblématique. Enfin, le troisième temps, consécutif à la Seconde Guerre mondiale, est celui de la métropole chaotique et en crise. Ce n'est plus le politique qui fait alors office de paravent de l'économie, mais c'est bien l'économie qui soumet l'architecture et le politique à ses desiderata. La ville-capitale perd ainsi ce que Jean Starobinski dénomme «son rôle emblématique », et ne conserve plus guère qu'un rôle symbolique.

\section{Les nouveaux traditionnalistes et la politique pendant la république de Weimar et le III Reich : l'architecte Paul Schmitthenner}

Wolfgang Voigt (architecte, historien de l'architecture à Francfort/Main)

L'une des principales figures de l'architecture allemande de l'entre-deux-guerres est Paul Schmitthenner, né en Alsace en 1884. Appartenant à la même génération que Le Corbusier, Walter Gropius et Mies van der Rohe, il est un enseignant charismatique au service du courant opposé : le nouveau traditionalisme. Il établit avec Paul Bonatz une nouvelle faculté au sein de l'École polytechnique de Stuttgart, qui devient pendant vingt ans l'école la plus populaire d'Allemagne, hostile à l'académie et fondée sur une vision morale de l'artisanat, le régionalisme et la tradition.

En revanche, sa cité-jardin de Staaken à côté de Berlin est un projet innovant en matière de standardisation, et les charpentes préfabriquées de Schmitthenner rivaliseront avec les logements sociaux des modernes. En opposition à la « Nouvelle Objectivité » de ces derniers, il élabore sa théorie de la « forme bâtie », établie sur un lien intime entre matériau, détail et expression architecturale.

En 1933, Schmitthenner aspire à devenir l'un des principaux architectes du nouveau régime avec autorité sur l'enseignement. Mais, bien que ses disciples fassent carrière dans l'administration nazie, ses espérances sont vaines. Conservant sa chaire à Stuttgart, il se replie dans une sorte d'exil interne à l'Allemagne, se déclarant vivement hostile à la rhétorique monumentale d'Albert Speer.

\section{Le plan de Moscou de 1935 : goûts et actions du décideur politique}

Elisabeth Essaïan (architecte, maître-assistante à l'ENS d'architecture de ParisBelleville)

Le 10 juillet 1935, Joseph Staline et Viatcheslav Molotov signent la décision portant adoption du plan général de la reconstruction de Moscou. Élaboré entre 1932 et 1935 par un collectif d'architectes et d'ingénieurs dirigé par l'urbaniste Vladimir Semionov, puis par l'architecte Sergueï Tchernychev, ce plan servira de modèle pour d'autres villes de l'URSS et, après 1945, dans le bloc oriental.

Les appellations communément utilisées pour désigner ce projet et les productions architecturales qui en sont issues - le plan lui-même, les bâtiments de grande hauteur, les immeubles d'appartements staliniens - insistent, à travers la forme adjective, sur le rôle de Staline, surnommé par ailleurs le « génial zodtchi [architecte] de Moscou ».

La réalité de cette « verticale du pouvoir » est mise en perspective à travers l'étude des formes, des lieux d'expression et des constructions révélant le goût et l'action du décideur politique. Plus qu'une mise en cause des effets de la doctrine du réalisme « socialiste », il s'agit de mettre en évidence la complexité des relations et des prises de décisions intervenues de 1932 à 1954 entre la sphère politique et le monde professionnel. 


\section{Les horizons politiques de l'architecture : l'action d'Oscar Niemeyer d'une dictature à l'autre (1937-1964)}

Margareth Pereira (architecte, professeure à l'université fédérale de Rio de Janeiro)

Le parcours d'Oscar Niemeyer est indissociable de la politique. Dans la chronologie assez linéaire de son œuvre, les commandes et les chantiers publics et institutionnels sont nombreux, du ministère de l'Éducation à Rio de Janeiro à la construction de Brasilia et au-delà. En dépit de sa cohérence apparente, elle révèle des formes d'action différentes quant au processus de conception et de réalisation et décline ainsi des manières changeantes de penser et pratiquer la politique.

L'action d'Oscar Niemeyer, de la dictature de Getúlio Vargas (1937-1945) à celle des militaires (1964-1988), permet d'engager plusieurs chantiers de recherche sur l'architectural et la politique à travers l'analyse de quatre commandes auxquelles Oscar Niemeyer a dû répondre : l'église de São Francisco à Pampulha, le siège des Nations unies à New York, le siège du Parti communiste français et le palais d'Itamaraty à Brasilia.

Sont ainsi examinés les conditions de possibilité de ces projets comme œuvre collective et individuelle, le jeu entre autonomie et hétéronomie qu'elles révèlent, la puissance et les impuissances de la pratique architecturale et enfin les intelligences et mésintelligences politiques, administratives, techniques, formelles qui s'y rencontrent.

\section{Jaap Bakema et la société ouverte : architecture, démocratie et l'État-providence aux Pays-Bas}

Dirk van den Heuvel (architecte, professeur associé à l'université technique de Delft)

Pendant tout l'après-guerre, l'architecte néerlandais Jaap Bakema a cherché à édifier une société démocratique et égalitaire faisant de la diversité des modes de vie le point de départ de son urbanisme. Cette position est formulée dans ses nombreuses interventions dans l'enceinte des CIAM, à commencer par la déclaration qu'il fit en 1947 au nom des jeunes architectes de son pays lors du congrès de Bridgwater, en Angleterre. C'est au congrès d'Otterlo qu'il introduisit en 1959 le thème de la société ouverte dans les discussions des CIAM et du Team 10. Il poursuivit son propos pendant toute sa vie, particulièrement dans ses échanges avec ses collègues américains et japonais.

Pour Bakema, la société ouverte correspondait à un projet social de changement, de contestation et de communication. Elle devait trouver ses fondations dans un Étatprovidence étendu à travers l'interaction entre les institutions gouvernementales, les citoyens et l'industrie. Pour lui, les Pays-Bas devaient tendre à devenir la société ouverte par excellence.

\section{Portugal 1974 : la révolution des CEillets et le SAAL; une relation triangulaire entre politique, processus et projet}

Nuno Grande (architecte, professeur associé à l'université de Coimbra)

La révolution portugaise du 25 avril 1974 a stimulé les attentes populaires quant à de meilleures conditions de vie. Les premiers gouvernements révolutionnaires ont tenté de répondre à cette urgence sociale en engageant des programmes publics comparables à ceux qui furent menés en Europe pendant les vingt années précédentes. 
Cette conjugaison entre modernité et postmodernité, pouvoir et contre-pouvoir, culture et contre-culture représente l'un des aspects les plus fascinants du processus révolutionnaire portugais.

Le SAAL ou Serviço de Apoio Ambulatório Local créé en juillet 1974 par l'architecte Nuno Portas, secrétaire au Logement du premier gouvernement provisoire, illustre cette conjugaison dans ses fragilités. Le programme visait à apporter une réponse rapide à la demande des associations d'habitants, à l'aide de «brigades » interdisciplinaires réunissant architectes, sociologues, juristes et travailleurs sociaux.

À Porto, ces brigades coordonnées par l'architecte Alexandre Alves Costa ont agi pour maintenir la population pauvre dans le centre, en réaction contre les programmes antérieurs visant à détruire les quartiers ouvriers et à transférer leurs habitants en périphérie. Engagés dans le processus révolutionnaire, des architectes de cette ville tels qu'Álvaro Siza, Pedro Ramalho et Sérgio Fernandez ont su insérer les nouveaux logements dans la structure urbaine existante, assurant ainsi à la population le « droit à la ville » cher à Henri Lefebvre.

\section{Les grands projets architecturaux « en l'État 》 (1958-2018)}

François Chaslin (architecte, écrivain, professeur honoraire des écoles d'architecture)

L'intervention explore une spécificité française qui a beaucoup étonné dans les années 1980 : la conduite d'opérations architecturales particulières, dites "grands projets », placées sous l'autorité directe, l'autorité « régalienne », des présidents de la République. Elle n'évoque qu'à peine les projets mitterrandiens pour saisir le phénomène sur une plus longue durée: six décennies, au cours desquelles se succédèrent huit présidents, trois maires de Paris et 24 gouvernements et majorités politiques issus des élections législatives.

\section{COURS À L'EXTÉRIEUR}

\section{Institute of Fine Arts de la New York University}

Printemps 2018 : Le Corbusier and the poetics of the machine age (cours magistral) et Territories of intertextuality: A transurban history of city form (séminaire).

\section{École d'architecture de l'université de Princeton}

Printemps 2018 : Frank Gehry's path : Art, technology, urbanity (séminaire).

\section{RECHERCHE}

\section{L'ARCHITECTURE DE FRANK GEHRY}

Une recherche approfondie a été engagée sur le déploiement de la démarche de projet de l'architecte américain du milieu des années 1950 à nos jours, fondée sur l'analyse détaillée des archives de l'agence - dessins, correspondances, photographies de maquettes et de chantiers, articles de presse -, et sur la visite des édifices. L'activité de Frank Gehry est inscrite à la fois dans son cadre culturel et social - la métropole de Los Angeles -, et dans les transformations qu'a connues l'architecture mondiale 
depuis la crise fatale du Mouvement moderne intervenue dans les années 1960. Un catalogue raisonné des quelque 12000 croquis d'étude conservés par l'agence, répartis en huit volumes, sera publié au cours des années à venir.

\section{L'AMÉRICANISME DANS L'ARCHITECTURE ET L'URBANISME DE LA RUSSIE}

Dans le cadre du phénomène d'ensemble de l'américanisme, ce système de transferts culturels déterminant de la modernité et de la modernisation, la relation la plus paradoxale est celle qui a relié la Russie et les États-Unis entre le dernier tiers du XIX ${ }^{\mathrm{e}}$ siècle et la seconde moitié du XX $\mathrm{X}^{\mathrm{e}}$ siècle. Chaque conjoncture dans l'histoire russe a été marquée par des représentations spécifiques des techniques, des territoires, de l'architecture et de la culture matérielle de l'Amérique. Une interprétation d'ensemble de ce phénomène est entreprise, à travers le prisme de l'architecture et de l'urbanisme, passant par une analyse serrée des discours, des projets et des édifices, inscrits dans leurs déterminations politiques, économiques et techniques, tout autant que dans la chronique de la littérature et celle du cinéma.

\section{ARCHITECTURE ET URBANISME DE LA FRANCE DE VICHY}

À la suite du cours donné au Collège de France au printemps 2016 et du colloque qui l'a conclu, la préparation d'un ouvrage collectif intitulé Architecture, arts et culture dans la France de Vichy, 1940-1944 a été entreprise.

Sur la base des recherches engagées depuis plusieurs années, un projet d'exposition a été élaboré, dont la présentation pourrait intervenir en 2020. L'identification des principales œuvres susceptibles d'être exposées a été engagée dans les fonds d'archives parisiens et régionaux.

\section{CASABLANCA, HISTOIRE ET PATRIMOINE}

Sur la base de recherches conduites entre 1989 et 2002 sur l'histoire de la forme urbaine et de l'architecture de Casablanca, qui ont abouti à des publications significatives, une recherche portant sur les stratégies de patrimonialisation a été engagée sur deux fronts distincts. Le premier est le repérage des processus en cours dans la ville quant à l'usage des quartiers et des édifices existants, à travers les changements de fonctions et de destinataires. Le second porte sur les politiques de préservation elles-mêmes, au moyen de la préparation de plans d'intervention, de règlements susceptibles d'entrer dans le droit de l'urbanisme, et dans la perspective de la constitution d'un dossier d'inscription sur la liste du patrimoine mondial de l'Unesco.

\section{PuBLiCATIONS}

\section{OUVRAGES}

COHEN J.-L. (dir.), L'Architecture entre pratique et connaissance scientifique, Paris, Éditions du Patrimoine, 2018.

COHEN J.-L. et CIORRA P. (dir.), Gli architetti di Zevi. Storia e controstoria dell'architettura italiana 1944-2000, Rome/Macerata, MAXXI/Quodlibet, 2018. 
COHEN J.-L., Architecture de l'avant-garde russe. Dessins de la collection Sergueï Tchoban, Paris, Beaux-Arts de Paris éditions, 2017.

COHEN J.-L., Architecture, modernité, modernisation, Paris, Collège de France/Fayard, coll. «Leçons inaugurales du Collège de France », vol. 265, 2017 ; édition numérique : Collège de France, 2017, DOI : 10.4000/books.cdf.4864 ; en ligne : https://books.openedition. org/cdf/4864.

\section{Préfaces et CONTRIBUtions À DES OUVRAGES COLleCtifS}

CoHEn J.-L., « Taliesin-France », in A.-L. Sol (dir.), Hervé Baley \& Dominique Zimbacca, architectes. Pour une autre modernité, Lyon, Lieux-Dits, 2018, p. 4-5.

COHEN J.-L., « Memory erased/regained: Marseille at war », in M. ENGEL (dir.), Fragments: Identity in Times of Conflict: A Programme of Lectures and Exhibitions: December 2015 to May 2017, BSR Architecture Programme, Rome, British School in Rome, 2018, p. 54-57.

COHEN J.-L., «Autour de la notion de "mémoire collective" de Maurice Halbwachs, et son appropriation par les urbanistes et architectes », in A. BERTHOZ et J. SCHEID (dir.), Les Arts de la mémoire et les images mentales, Paris, Collège de France, coll. « Conférences », 2018, p. 121-132, DOI : 10.4000/books.cdf.5434 ; en ligne : https://books.openedition.org/cdf/5534.

COHEN J.-L., « The exhibition pavilion for Theodor Ahrenberg, or Le Corbusier between the collection and the museum », in C. PILTO (dir.), Living with Matisse, Picasso and Christo, Theodor Ahrenberg and his Collections, Londres, Thames \& Hudson, 2018, p. 132-135.

COHEN J.-L., «Zevi sotto Zevi », in J.-L. COHEN et P. CIORRA (dir.), Gli architetti di Zevi; storia e controstoria dell'architettura italiana 1944-2000, Rome/Macerata, MAXXI/ Quodlibet, 2018, p. 33-40.

COHEN J.-L., « Berber brutalism and the grammar of reconstruction, conversation with Yto Barrada », in L. JoHnSON (dir.), Yto Barrada, Londres, Barbican, 2018, p. 31-43.

COHEN J.-L., «Le Corbusier's architectural œuvre, or surprise as a strategy », in D. Pauly (dir.), Le Corbusier, the Paths to Creation, Séoul, Seoul Arts Center, 2017, p. 82-90.

COHEN J.-L., «A lost vanguard discovered », in D. CHUnG (dir.), Architecture as Movement: Modern Architecture in South Korea, 1987-1997, Séoul, National Museum of Modern and Contemporary Art, 2018, p. 84-96.

COHEN J.-L., «Verso una storia transurbana delle città », in G. MEnNA (dir.), Historia Rerum. Scritti in onore di Benedetto Gravagnuolo, Naples, CLEAR, 2017, p. 150-157.

COHEN J.-L., « Entretien avec Elisabeth Essaïan », in A. CRICONIA et E. EsSAÏAN (dir.), Lina Bo Bardi. Enseignements partagés, Paris, Archibooks, 2017, p. 238-253.

COHEN J.-L., « L'encyclopédie et le palimpseste », in R. RECHT et J. PIJAUDIER-CABOT (dir.), Laboratoire d'Europe, Strasbourg 1880-1930, Strasbourg, Musées de la ville de Strasbourg, 2017, p. 34-43.

COHEN J.-L., « Architecture et forme urbaine », Annuaire du Collège de France 2015-2016. Résumé des cours et travaux, $\mathrm{n}^{\circ} 116,2018$, p. 537-547, DOI : 10.4000/annuaire-cdf.13124 ; en ligne : https://journals.openedition.org/annuaire-cdf/13124.

\section{ARTICLES DE PÉRIODIQUES}

COHEN J.-L., « Frank Gehry. Studio e residenza Danziger. Los Angeles, 1964-1965 », Domus, vol. 1020,2018 , p. 22-31. 